\title{
Sending messages in moving cells: mRNA localization and the regulation of cell migration
}

DOI:

10.1042/EBC20190009

\section{Document Version}

Accepted author manuscript

Link to publication record in Manchester Research Explorer

\section{Citation for published version (APA):}

Herbert, S., \& Costa, G. (2019). Sending messages in moving cells: mRNA localization and the regulation of cell migration. Essays in biochemistry. https://doi.org/10.1042/EBC20190009

\section{Published in:}

Essays in biochemistry

\section{Citing this paper}

Please note that where the full-text provided on Manchester Research Explorer is the Author Accepted Manuscript or Proof version this may differ from the final Published version. If citing, it is advised that you check and use the publisher's definitive version.

\section{General rights}

Copyright and moral rights for the publications made accessible in the Research Explorer are retained by the authors and/or other copyright owners and it is a condition of accessing publications that users recognise and abide by the legal requirements associated with these rights.

\section{Takedown policy}

If you believe that this document breaches copyright please refer to the University of Manchester's Takedown Procedures [http://man.ac.uk/04Y6Bo] or contact uml.scholarlycommunications@manchester.ac.uk providing relevant details, so we can investigate your claim.

\section{OPEN ACCESS}


Sending messages in moving cells:

mRNA localization and the regulation of cell migration

Shane P. Herbert ${ }^{1}$ and Guilherme Costa ${ }^{1 *}$

${ }^{1}$ Faculty of Biology, Medicine and Health, The University of Manchester, Manchester, M13 9PT, UK

${ }^{*}$ Correspondence:

guilherme.costa@manchester.ac.uk 


\section{Abstract}

Cell migration is a fundamental biological process involved in tissue formation and homeostasis. The correct polarization of motile cells is critical to ensure directed movement and is orchestrated by many intrinsic and extrinsic factors. Of these, the subcellular distribution of mRNAs and the consequent spatial control of translation are key modulators of cell polarity. mRNA transport is dependent on cis-regulatory elements within transcripts, which are recognized by trans-acting proteins that ensure the efficient delivery of certain messages to leading cell edge of migrating cells. At their destination, translation of localized mRNAs then participates in regional cellular responses underlying cell motility. In this review, we summarize the key findings that established mRNA targeting as a critical driver of cell migration and how the characterization of polarized mRNAs in motile cells has been expanded from just a few species to hundreds of transcripts. We also describe the molecular control of mRNA trafficking, subsequent mechanisms of local protein synthesis and how these ultimately regulate cell polarity during migration.

\section{Introduction}

Cell migration is a complex and dynamic process implicated in a variety of biological phenomena that shape, maintain and remodel multicellular organisms. Deciphering principles of cell motility has thus contributed to our fundamental understanding of a wide range of tissue processes, ranging from morphogenesis [1] and immune surveillance [2] to wound healing [3] and tumor invasion [4]. Migrating cells are polarized along their directional axis, displaying a highly active leading edge that extends actin-rich protrusions to sense environmental cues and a rear that can steer movement, propel the cell forward or lead other cells (Figure 1A) [5, 6]. The polarized distribution of a vast number of proteins within migrating cells and their localized roles in orienting cell motility is well established [7]. Although less understood, messenger RNA (mRNA) localization is also highly polarized in migrating cells and is increasingly becoming the focus of much attention for its similar ability to coordinate cell motility.

The subcellular distribution of mRNAs and their local translation are tightly 
regulated steps between gene transcription and protein activity [8]. This mode of spatial control of gene expression is an evolutionary conserved mechanism critical for the establishment of cell polarity across the living world [9]. Indeed, studies focusing on the function of targeted transcripts have proposed several advantages conferred by mRNA localization (Figure 1B). Firstly, local synthesis of multiple protein copies from a single mRNA may be more cost-effective than transporting each individual protein. Second, spatially restricted translation of localized mRNAs increases local protein concentrations to enhance outcomes. Third, newly synthesized proteins may undergo local posttranslational modifications that renders them functionally distinct form pre-existing ones. Fourth, members of protein complexes can be locally co-synthesized to facilitate complex formation. Finally, local pools of readily available mRNA for translation may contribute to rapid and regionalized cellular functions if translation is coupled to dynamic environmental stimuli. While some of these premises have been founded on indirect biological evidence, systematic analysis strongly supports key benefits for such spatiotemporal control of protein synthesis, particularly in defining the fidelity and sensitivity of protein function [10]. Indeed, the authors of this study suggested that incorrect mRNA targeting might alter the location of newly translated products leading to ectopic protein activity, which in turn may be the mechanistic basis of human pathologies [10]. In the context of cell motility, to date, hundreds of mRNAs have been shown to display patterns of asymmetric distribution in migrating cells [11-16]. Furthermore, the localization of some mRNA species has been implicated in shaping the molecular mechanisms orchestrating directed cell movement [17-19]. Here, we review the key findings that uncovered key roles for polarised mRNA distribution in motile cells. Moreover, we discuss how mRNAs are targeted and how their local translation ultimately functions to regulate cell migration in distinct settings.

\section{mRNA localization in migrating cells: where and why?}

The ability of motile cells to navigate through surrounding environments is tightly regulated by dynamic membrane remodeling and adhesion to the substrate. Early evidence indicating active transcript localization during these 
keys cellular events suggested that mRNAs could participate in cell migration. While particular transcripts demonstrated active polarization in migrating cells towards the leading edge [20], RNA binding proteins (RBP) were later implicated at early stages of cell adhesion [21]. Furthermore, mRNAs were shown to be recruited to focal adhesions (FA) [22], protein complexes formed at the cell periphery that link the cytoskeleton to the extracellular matrix and promote forward movement [23]. Despite the fact that the precise function of targeting mRNAs to adhesion sites in migrating cells remains poorly understood, a solid body of literature focusing either on single transcripts or on transcriptome-wide approaches, has since shed light on the role played by mRNA localization during cell migration. Moreover, a number of remarkable technological advances in the visualization of individual transcripts in fixed and live cells (Box 1) [24] have proven to be invaluable resources in understanding the subcellular distribution, dynamics and function of mRNAs in motile cells.

\section{Cytoskeleton remodelers encoded by localized mRNAs}

The subcellular localization of mRNAs encoding cytoskeleton remodelers has received particular attention due to the clear roles played by the encoded proteins in the control of cytoskeletal dynamics [25]. Reports dedicated to cytoskeletal transcripts have strengthened the idea that their local translation enhances rapid morphological responses to extracellular cues during membrane remodeling. In particular, spatial sorting of $\beta$-actin mRNA is a wellstudied example of a transcript whose localization has received particular attention in the context of cell migration. Early studies showed that chemoattractant signals, such as lysophosphatidic acid (LPA) and serum, induce a rapid polarization of $\beta$-actin mRNA towards the leading edge of motile cells. Transcript translocation in this context is dependent on the RhoA/Rho kinase signaling axis and the subsequent activity of the motor protein myosin IIB $[26,27]$. This work led to an original model in which $\beta$-actin mRNA travels along microfilaments with the aid of motor proteins to drive it's polarized accumulation. Further detailed analysis employing reporter tools has, nevertheless, unveiled that $\beta$-actin mRNAs display behavior patterns not only characteristic of motored transport, but also of diffusive movement [28, 29]. These observations have raised questions regarding the role of cytoplasmic 
flow in $\beta$-actin localization, possibly regulated by myosin IIB contractility, and the coordination between distinct types of mRNA trafficking routes [25]. Once reaching their destination, $\beta$-actin mRNAs are bound by the translation

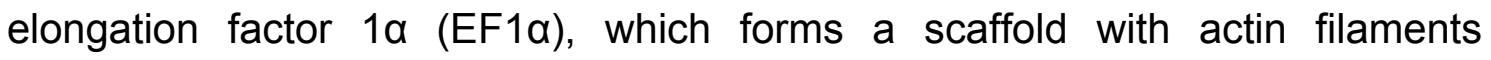
responsible for anchoring transcripts to the cell cytoskeleton (Figure 2) [30]. This way, the transport of $\beta$-actin mRNAs and their accumulation at the leading edge maintains cell polarity and ensures persistent directionality during migartion $[31,32]$. This is presumably achieved through increased concentrations of newly synthesized $\beta$-actin monomers, which in turn have less affinity for capping and severing proteins [33] and more likely to contribute to filament polymerization and active membrane remodeling [34]. Such hypothesis has been supported by genetic manipulation experiments demonstrating that tethering $\beta$-actin mRNA to FA protein complexes enhances cell adhesion growth and stability [17].

The actin-related protein 2/3 (ARP2/3) complex is a critical actin nucleator important for the creation of branched actin networks and the consequent support of protrusion formation. Like the ARP $2 / 3$ complex, ecoding $A R P 2 / 3$ transcripts are localized at the leading edge during migration [35]. This subcellular pattern of mRNA distribution is stimulated by external signaling cues and it is dependent on both microfilament and microtubule networks [35, 36]. Furthermore, loss of cell directionality caused by knockdown depletion of the subunit ARP2 can only be rescued when ARP2 mRNA is targeted to protrusions but not to perinuclear domains [18]. Intriguingly, all seven subunits that compose ARP2/3 are encoded by mRNAs targeted to fibroblast protrusions [35]. Hence, ARP2/3 mRNAs constitute a likely example of co-localized transcripts whose local translation could facilitate the regionalized assembly of protein complexes without the need to translocate individual subunits, although experimental evidence for this is currently lacking. Similarly to $\beta$-actin and $A R P 2 / 3$, the transcript Cofilin1 also accumulates at the cell front of motile cells. [19]. Cofilin1 encodes a member of the actin depolymerization factor/cofilin family of cytoskeleton remodeling proteins that sever pre-existing actin filaments and destabilize branched networks [37]. Interestingly, Cofilin1 localization mechanisms are reminiscent of those responsible for targeting $\beta$ actin and like ARP2 mRNAs, reduced cell directionality caused by the loss of 
Cofilin1 protein is restored with the correct targeting of exogenous Cofilin1 transcripts to cell protrusions but not elsewhere in the cell [19]. The remarkable similarities in spatial distribution displayed by $\beta$-actin, ARP2/3 and Cofilin1 are suggestive of conserved localization mechanisms, which could be broadly shared amongst mRNAs coding for proteins involved in common cellular processes. Global profiling of localized mRNAs has now tried to address this aspect in several cell types [11-16]. But whether and how migrating cells coregulate the transport and the local translation of these mRNAs at sites of active cytoskeletal remodeling is yet to be understood.

The broader cell protrusion transcriptome

Although the localization of mRNAs to the front of motile cells is a longaccepted phenomenon, the true global extent of this process was only revealed upon employment of modified Boyden chambers [38] to fractionate protrusions from cell bodies (Table 1). In combination with whole transcriptome profiling, this method has now allowed the identification of hundreds of mRNAs preferentially localized to the leading edge of fibroblasts and invasive tumor cells [11-16]. Many mRNA species are consistently enriched in protrusions of distinct cell types, indicating that their localization likely reflects roles in common pathways underlying cell migration. Interestingly, whilst many of these mRNAs encode proteins with implications in membrane dynamics and cell locomotion, a remarkable proportion of protrusion-enriched transcripts code for ribosomal proteins. Although far from being understood, the latter has been observed in distinct polarized cells and prompted some authors to hypothesize localized de novo ribosome assembly and repair [39]. Notably, $\beta$-actin, Arp2 and Cofilin1 are not consitently identified in these studies, suggesting that key localization mechanisms may differ between assays and cell types.

Using mouse fibroblasts, Mili et al. elegantly reported that some mRNAs identified in cell protrusions, such as RAB13, PKP4 and $A N K R D 25$, are associated with plus ends of detyrosinated (stable) microtubules [11]. More recently, the same author described how the stiffness of the cell substrate dictates the composition of the localized mRNAs. Increased Rho signaling and actomyosin contractility induced by stiff substrates was reported to drive localization of transcripts encoding cell morphology regulators to large cell 
protrusions. mRNAs present in smaller filopodia-like structures encode mostly ribosomal proteins, which do not depend on substrate-induced cell contractility [15].

The profiling of mRNAs present at the leading front of tumor cells has been part of the quest to dissect mechanisms of metastatic spreading. Thus, several invasive cell models have been used with the intent to link mRNA localization to metastasis. Transcripts identified in cellular protrusions of Moloney sarcoma virus-MDCK-invasive cells were exploited to elucidate how Rho signaling and mysosin IIB regulate the protrusion localization of mRNAs encoding signaling molecules involved in cell adhesion and migration, such as M-RAS and SHP2 [12]. Metastatic breast cancer cells on the other hand have been employed to illustrate that metastatic and non-metastatic cell protrusions not only contain distinct levels of particular mRNAs, but also different enrichments for specific mRNA isoforms, like the cell junction regulator PKP4 [13]. In a comparative transcriptomic and proteomic study, a cohort of mRNAs and their encoded proteins were found to be enriched in protrusions of prostate, breast, fibrosarcoma and glioma cell lines with metastatic properties. Some of these transcripts encode proteins implicated in tumor progression, including AHNAK, Septin9a and eIF4E, and while their localized roles was not addressed, protein knockdown reduced metastatic cell motility and invasion [14]. Hence, the wide spectrum of protrusion-targeted mRNAs suggests diverse as yet unknown functional roles for local protein translation in the coordination of cell migration.

\section{mRNA targeting mechanisms}

Localized mRNAs are usually transported to their destination in large ribonucleoprotein (RNP) complexes formed with RBPs [40]. The association between mRNAs and RBPs is a pivotal posttranscriptional process that regulates the transcript life cycle, from nuclear export and localization to translation and decay. RNP biology goes beyond the scope of this review and we refer readers to excellent reviews published on this subject [41-43]. RBPs acting as trans-acting factors in the spatial targeting of mRNAs integrate RNP transport complexes, either in the nucleus soon after transcription or in the cytoplasm upon nuclear export. Once in the cytoplasm, RNP transport along the 
cytoskeleton orchestrated by motor proteins is responsible for their polarized delivery [44]. RNPs present at the cell periphery take part in initial steps of cell spreading and in this respect, their localized function has been linked to cell adhesion [21]. Cis-regulatory elements, also known as zipcodes, contained within transported transcripts dictate the interaction of mRNAs with RBPs, and ensure correct mRNA targeting. Zipcodes vary between a few nucleotides in length to several hundred and are formed either by specific mRNA primary sequences, secondary/tertiary mRNA structures or a combination of both [45, 46]. This level of complexity has rendered the precise mapping of zipcodes and the characterization of mRNA-RBP binding specificities a difficult task. For the same reason, this has hindered the identification of conserved zipcodes that drive protrusion targeting. Thus, functional testing of the disruption of localized mRNAs in migrating cells has been arduous. Nonetheless, several studies have now provided significant contributions to our knowledge of the roles played by particular cis- and trans-acting determinants in transcript localization within motile cells and how mRNA-RBP interactions ultimately modulate cell migration.

\section{Cis-regulation of mRNA transport}

Whilst zipcode sequences have been defined throughout transcript bodies, the majority of reported examples confine such elements to 3' untranslated regions (UTR). Likewise, the distribution of many protrusion-localized mRNAs in motile cells has been attributed to zipcode-containing 3'UTRs [11, 15, 18, 19, 47]. However, $\beta$-actin is the only example of a protrusion-targeted mRNA whose 3'UTR zipcode has been precisely characterized. Hence, the $\beta$-actin zipcode is now a well-established paradigm for the cis-regulation of mRNA transport in several biological contexts, and is the best studied model of mRNA targeting during cell migration. Comprehensive analysis of the $\beta$-actin 3' UTR using reporter constructs concluded that the zipcode is composed of a 54-nucleotide sequence sufficient for transcript localization at the cell front [47]. Furthermore, targeting the zipcode with antisense oligonucleotides to prevent the interaction with trans-acting factors disrupts cell polarity and reduces persistence of cell directionality $[31,47,48]$, confirming that cis-regulation of $\beta$-actin localization controls migration. Intriguingly, contradicting evidence has favored transcript diffusion through the microstructure of the cytoplasm over the function of its 
targeting zipcode as the main determinant of $\beta$-actin mRNA localization [49]. Together with endogenous transcript tracking experiments describing diffuse $\beta$ actin movement $[28,29]$, this again implicates the cytoplasmic flow of motile cells in the control mRNA movement. However, it is important to notice that many of these conclusions have been based on exogenous reporter transcripts, which might not truly mimic the behavior of endogenous mRNAs. As such, continued development of new gene editing tools will be critical for the future dissection of endogenous zipcodes to define roles in mRNA localization.

Whether similar or conserved cis-regulatory sequences mediate co-transport of multiple mRNAs to the leading front of motile cells is not known, but two reports have now shed new light on this idea. Firstly, Wang et al. developed a competition assay that evaluated how overexpression of several 3'UTRs of protrusion-localized mRNAs resultes in the mislocalization of unrelated endogenous transcripts and affected cell migration. The authors suggested that exogenous 3'UTRs saturate complexes co-trafficking groups of endogenous mRNAs by presumably outcompeting their RBP-mediated transport [15]. Secondly, Hotz et al. used Dictyostelium cells to investigate the simultaneous transport of chemotactic pathway mRNAs to the cell front. Interestingly, the 3'UTRs of some of these transcripts comprise similar cis-regulatory elements recognized by the RBP Pumilio. The authors further showed that in contrast to intact sequences, reporter mRNAs containing mutations in the Pumilio binding sites fail to display correct patterns of spatial distribution [50]. Hence, distinct 3'UTR motifs may play conserved roles in the protrusion-targeting of diverse mRNAs during cell motility, although functional evidence remains elusive.

\section{Delivery of mRNAs by trans-acting regulators}

The characterization of the $\beta$-actin zipcode led to the discovery of an RBP responsible for its mRNA localization in motile cells, the zipcode binding protein 1 (ZBP1) [51]. A great body of work has now been attributed to this trans-acting factor in targeting a variety of mRNAs during cell migration, and other functionalities that go beyond simple trafficking of $\beta$-actin mRNA. The reported involvement of ZBP1 in multiple cancer types, and association with varied prognosis, indicates that ZBP1-mediated posttranscriptional control of its target mRNAs may regulate disease progression and metastasis [52]. Indeed, ZBP1 
drives protrusion formation and cell migration of particular tumor cell models [53-56], whilst repressing invasiveness in others [57, 58]. Furthermore, ZBP1 has been recently identified as novel reader of the $N^{6}$-methyladenosine $\left(m^{6} A\right)$ RNA modification in cancer cells. Binding of ZBP1 to $\mathrm{m}^{6} \mathrm{~A}$-modified mRNAs modulates the translation of target transcripts and controls oncogenic functions, including cell invasion [59]. These intriguing findings raise the possibility that mRNA modifications might determine transcript localization to the leading front of motile cells. ZBP1 forms a complex with nascent $\beta$-actin transcripts in the cell nucleus [60,61], where two C-terminal RNA binding domains recognize spaced sequences within the zipcode and induce a conformational change in the mRNA molecule [62, 63]. To a lesser extent, ZBP1 is also understood to localize $A R P 2 / 3$ and Cofilin1 at the leading front of motile cells $[19,58]$, but whether these mRNAs are co-transported is not known. In the cytoplasm, ZBP1 acts as an adaptor protein that mediates motored transport of $\beta$-actin mRNAs along microtubules and microfilaments (Figure 2) [29, 60, 64]. Disruption of the adaptor-motor complex results in $\beta$-actin mislocalization and reduces directional cell movement [65]. Moreover, ZBP1 localizes $\beta$-actin adhesions regions in cell protrusions where it has been proposed to regulate FA stability [58]. Despite these findings, the ZBP1-mediated mRNA targeting of mRNAs in the context of tissue dynamics and disease remains rather unexplored.

Although less understood than ZBP1, the tumor suppressor protein adenomatous polyposis coli (APC) is another trans-acting factor with a critical role in mRNA localization during migration. APC binds to microtubules growing into areas of protrusion formation [66-68] and its interaction with plus end tracking proteins promotes microtubule stability and cell motility $[69,70]$. Initial evidence for its mRNA localization properties demonstrated that APC is a component of RNPs accumulated at the plus end of detyrosinated microtubules in cell protrusions [11]. Subsequently, this process was shown to depend on the physical properties of the extracellular environment through which cells navigate (Figure 2). Mechanotransduction signals couple the substrate stiffness to the detyrosinated microtubule network and the consequent APC-dependent localization of mRNAs [15]. APC-containing RNPs are also associated with the fused in sarcoma (FUS) protein [71], an RBP that can form toxic aggregates with links to human disorders [72]. Intriguingly, FUS mutations carried by 
amyotrophic lateral sclerosis patients lead to the disruption of microtubule detyrosination and hinder transcript targeting through APC, strongly connecting mechanisms of mRNA localization to disease [73]. Crosslinking technology proved the ability of APC to bind the 3' UTRs of a large number of transcripts, many of which encode cytoskeletal regulators [74]. Three putitive mRNA motifs seem to be overrepresented in APC-bound mRNAs, but whether APC directly interacts with these primary sequences and/or secondary structures, and whether other RBPs promote these interactions to drive mRNA targeting, are themes yet to be explored.

\section{On-site protein synthesis and the control of cell migration}

Translation of transcripts accumulated at the leading edge has been generally regarded as the ultimate purpose of localized mRNAs in motile cells [25]. While control of protein synthesis at sites distant from cell bodies is a well-accepted phenomenon, mechanisms of local translation during migration are less understood than those mediating mRNA targeting. Lessons from axon path finding have elucidated how local translation is controlled by external stimuli and can be extrapolated to general mechanisms of cell movement [39]. Attractive and repulsive cues guide the leading front of axons, or the growth cone, as they grow towards the targeted destination [75]. These signaling molecules elicit the local translation of proteins responsible for rapid cytoskeletal remodeling and, thereby, direct growth cone steering [39]. For example, directional exposure to the attractive proteins Netrin1 and the brainderived neurotrophic factor (BDNF) results in a polarized inhibition of translation repressors and asymmetric synthesis of $\beta$-actin towards the signal $[76,77]$. In contrast, repulsive cues such as Sema3A and Slit2 induce translation events of RhoA and Cofilin1, respectively, and the consequent cytoskeletal disassembly events necessary to steer the growth cone away from the signal $[78,79]$. However, whether this reflects precise spatial control of distinct transcript translation or broader global protein synthesis remains unclear.

The regulation of translation by RBPs trafficking mRNAs to sites of protein production is itself fundamental for the control of asymmetrical protein production and it requires particular attention. In the case of $\beta$-actin traficking, 
the high affinity of ZBP1 to its target maintains the transcript in a repressed state to prevent protein synthesis during transport [80]. ZBP1 phosphorylation downstream of signals such as BDNF through local Src kinase activity alleviates the binding to $\beta$-actin and allows its translation (Figure 2) [77, 80]. Localized translation of transcripts transported by APC, on the other hand, is regulated by FUS (Figure 2). Depletion of the latter from APC-containing RNP complexes reduces the translation of mRNAs targeted to cell protrusions [71].

Although widely assumed to be the case, whether local protein synthesis directly contributes to the regionalized cellular processes of signal sensing, protrusion formation and adhesion is not yet entirely resolved. However, some reports provide robust evidence that local translation is tightly associated with the biological events underlying cell migration. Willet et al. demonstrated that translational machinery components and newly synthesized proteins are present at the leading edge of fibroblasts and co-localize with FAs [81, 82]. In addition, Katz et al. imaged ZBP1-transported $\beta$-actin to FA regions, where the increased mRNA dwelling times indicate that these transcripts undergo active transcript translation [58]. Similarly, later studies used simultaneous tracking of single mRNAs and ribosomes to propose that $\beta$-actin is preferentially translated when in close proximity to FAs [83]. Finally, Mardakheh et. al. devised a strategy to locally repress protein synthesis in metastatic breast tumor cells. Upon exposing the leading edge to translation inhibitors, migrating cells exhibit reduced protrusion formation and invasion in vitro [16]. In summary, there is compelling evidence that local protein synthesis of specific mRNAs is likely to be critical for cell protrusion formation and motility, although direct evidence is still lacking.

\section{Future perspectives}

More than three decades of research has established that the spatial control of gene expression is critically implicated in fundamental aspects of cell migration. Genomic studies have unveiled a vast variety of mRNAs present at the leading front of motile cells and have shed light on the mechanisms responsible for subcellular mRNA targeting. These have also demonstrated that the physical 
and chemical properties of the extracellular environment, transduced through the contractile cell machinery, shapes mRNA localization. Translation of localized mRNAs has been proposed to ensure rapid and regionalized responses necessary during cell migration. Nonetheless, the advantages that these mechanisms provide over protein localization have been rather indirectly inferred from the studies described in this review and elsewhere. Experimental limitations hinder deciphering precise mechanisms controlling mRNA localization and make it difficult to fully understand the extent to which it regulates cell migration in complex environments. In fact, whether mRNA trafficking within motile cells contributes to tissue formation in vivo or if it has implications in tumor invasion and cancer progression have yet to be unveiled. Emerging technology will undoubtedly bring new insights into these unanswered questions. The continuous development of sophisticated imaging tools is allowing the visualization of mRNA localization and local translation with increasing levels of detail (Box 1). Furthermore, novel gene-editing methods open exciting avenues for the identification and characterization of mRNA cistrafficking mechanisms. Ultimately, the application of such strategies to in vivo cell migration models will expand our knowledge on how the spatial control of gene expression broadly modulates cell migration in tissue development and disease.

\section{Summary points}

- Motile cells accumulate a vast number of mRNAs in protrusions formed at their leading edge. The nature of the proteins encoded by some of these transcripts has suggested that the local availability of mRNA contributes to rapid and compartmentalized cell responses during migration.

- Transcripts localization is accomplished through the interaction between cis-regulatory elements contained within the mRNA molecule and transacting proteins. mRNA trafficking along the cytoskeleton is modulated by signaling cues and the physical properties of the environment. 
- Translation of localized mRNAs can be triggered by regional signals and dependent on RNA interacting proteins. Although not fully understood, protein synthesis at the leading front of motile cells is necessary for cell migration and it is likely to have critical roles in tissue development and disease.

\section{Competing interests}

The authors declare that there are no competing interests associated with the manuscript

\section{Abbreviations}

APC, adenomatous polyposis coli

ARP2/3, Actin-related protein 2/3

bDNA, branched DNA

BDNF, brain-derived neurotrophic factor

CRISPR, clustered regularly interspaced short palindromic repeats

EF1a, elongation factor $1 \alpha$

$F A$, focal adhesion

FISH-STIC, fluorescent in situ hybridization with sequential tethered and intertwined oligodeoxynucleotide complexes

$\mathrm{FP}$, fluorescent protein

FUNCAT-PLA, fluorescent noncanonical amino acid tagging

FUS, fused in sarcoma

$\mathrm{HCR}$, hybridization chain reaction

LPA, lysophosphatidic acid

$\mathrm{m}^{6} \mathrm{~A}, N^{6}$-methyladenosine

mRNA, messenger RNA

NCT, nascent chain tracking

$\mathrm{POI}$, protein of interest

RBP, RNA binding protein

RNP, ribonucleoprotein

seqFISH, sequential fluorescent in situ hybridization 
SINAPS, single-molecule imaging of nascent peptides

sm(i)FISH, single molecule (inexpensive) fluorescent in situ hybridization

TRICK, translating RNA imaging by coat protein knock-off

UTR, untranslated region

ZBP1, zipcode binding protein 1 


\section{References}

1. Reig, G., E. Pulgar, and M.L. Concha, Cell migration: from tissue culture to embryos. Development, 2014. 141(10): p. 1999-2013.

2. Moreau, H.D., et al., Integrating Physical and Molecular Insights on Immune Cell Migration. Trends Immunol, 2018. 39(8): p. 632-643.

3. Martin, P. and R. Nunan, Cellular and molecular mechanisms of repair in acute and chronic wound healing. Br J Dermatol, 2015. 173(2): p. 370-8.

4. Lintz, M., A. Munoz, and C.A. Reinhart-King, The Mechanics of Single Cell and Collective Migration of Tumor Cells. J Biomech Eng, 2017. 139(2).

5. Mayor, R. and S. Etienne-Manneville, The front and rear of collective cell migration. Nat Rev Mol Cell Biol, 2016. 17(2): p. 97-109.

6. Krause, M. and A. Gautreau, Steering cell migration: lamellipodium dynamics and the regulation of directional persistence. Nat Rev Mol Cell Biol, 2014. 15(9): p. 577-90.

7. Bryant, D.M. and K.E. Mostov, From cells to organs: building polarized tissue. Nat Rev Mol Cell Biol, 2008. 9(11): p. 887-901.

8. Ryder, P.V. and D.A. Lerit, RNA localization regulates diverse and dynamic cellular processes. Traffic, 2018. 19(7): p. 496-502.

9. Buxbaum, A.R., G. Haimovich, and R.H. Singer, In the right place at the right time: visualizing and understanding $m R N A$ localization. Nat Rev Mol Cell Biol, 2015. 16(2): p. 95-109.

10. Weatheritt, R.J., T.J. Gibson, and M.M. Babu, Asymmetric mRNA localization contributes to fidelity and sensitivity of spatially localized systems. Nat Struct Mol Biol, 2014. 21(9): p. 833-9.

11. Mili, S., K. Moissoglu, and I.G. Macara, Genome-wide screen reveals APC-associated RNAs enriched in cell protrusions. Nature, 2008. 453(7191): p. 115-9.

12. Stuart, H.C., et al., Localized Rho GTPase activation regulates RNA dynamics and compartmentalization in tumor cell protrusions. J Biol Chem, 2008. 283(50): p. 34785-95.

13. Jakobsen, K.R., et al., Direct RNA sequencing mediated identification of $m R N A$ localized in protrusions of human MDA-MB-231 metastatic breast cancer cells. J Mol Signal, 2013. 8(1): p. 9. 
14. Shankar, J., et al., Pseudopodial actin dynamics control epithelialmesenchymal transition in metastatic cancer cells. Cancer Res, 2010. 70(9): p. 3780-90.

15. Wang, T., et al., Extracellular matrix stiffness and cell contractility control RNA localization to promote cell migration. Nat Commun, 2017. 8(1): p. 896.

16. Mardakheh, F.K., et al., Global Analysis of mRNA, Translation, and Protein Localization: Local Translation Is a Key Regulator of Cell Protrusions. Dev Cell, 2015. 35(3): p. 344-57.

17. Katz, Z.B., et al., beta-Actin mRNA compartmentalization enhances focal adhesion stability and directs cell migration. Genes Dev, 2012. 26(17): p. 1885-90.

18. Liao, G., B. Simone, and G. Liu, Mis-localization of Arp2 mRNA impairs persistence of directional cell migration. Exp Cell Res, 2011. 317(6): p. 812-22.

19. Maizels, Y., et al., Localization of cofilin $m R N A$ to the leading edge of migrating cells promotes directed cell migration. J Cell Sci, 2015. 128(10): p. 1922-33.

20. Lawrence, J.B. and R.H. Singer, Intracellular localization of messenger RNAs for cytoskeletal proteins. Cell, 1986. 45(3): p. 407-15.

21. de Hoog, C.L., L.J. Foster, and M. Mann, RNA and RNA binding proteins participate in early stages of cell spreading through spreading initiation centers. Cell, 2004. 117(5): p. 649-62.

22. Chicurel, M.E., et al., Integrin binding and mechanical tension induce movement of mRNA and ribosomes to focal adhesions. Nature, 1998. 392(6677): p. 730-3.

23. Sun, Z., S.S. Guo, and R. Fassler, Integrin-mediated mechanotransduction. J Cell Biol, 2016. 215(4): p. 445-456.

24. Pichon, X., et al., A Growing Toolbox to Image Gene Expression in Single Cells: Sensitive Approaches for Demanding Challenges. Mol Cell, 2018. 71(3): p. 468-480.

25. Liao, G., et al., Control of cell migration through mRNA localization and local translation. Wiley Interdiscip Rev RNA, 2015. 6(1): p. 1-15. 
26. Latham, V.M., et al., A Rho-dependent signaling pathway operating through myosin localizes beta-actin mRNA in fibroblasts. Curr Biol, 2001. 11(13): p. 1010-6.

27. Latham, V.M., Jr., et al., Beta-actin mRNA localization is regulated by signal transduction mechanisms. J Cell Biol, 1994. 126(5): p. 1211-9.

28. Park, H.Y., et al., Visualization of dynamics of single endogenous $m R N A$ labeled in live mouse. Science, 2014. 343(6169): p. 422-4.

29. Fusco, D., et al., Single mRNA molecules demonstrate probabilistic movement in living mammalian cells. Curr Biol, 2003. 13(2): p. 161-167.

30. Liu, G., et al., Interactions of elongation factor 1alpha with F-actin and beta-actin mRNA: implications for anchoring mRNA in cell protrusions. Mol Biol Cell, 2002. 13(2): p. 579-92.

31. Shestakova, E.A., R.H. Singer, and J. Condeelis, The physiological significance of beta -actin mRNA localization in determining cell polarity and directional motility. Proc Natl Acad Sci U S A, 2001. 98(13): p. 704550 .

32. Park, H.Y., et al., An unbiased analysis method to quantify mRNA localization reveals its correlation with cell motility. Cell Rep, 2012. 1(2): p. 179-84.

33. Saha, S., et al., Arginylation regulates intracellular actin polymer level by modulating actin properties and binding of capping and severing proteins. Mol Biol Cell, 2010. 21(8): p. 1350-61.

34. Condeelis, J. and R.H. Singer, How and why does beta-actin mRNA target? Biol Cell, 2005. 97(1): p. 97-110.

35. Mingle, L.A., et al., Localization of all seven messenger RNAs for the actin-polymerization nucleator Arp2/3 complex in the protrusions of fibroblasts. J Cell Sci, 2005. 118(Pt 11): p. 2425-33.

36. Mingle, L.A., et al., LPA-induced mutually exclusive subcellular localization of active RhoA and Arp2 mRNA revealed by sequential FRET and FISH. Histochem Cell Biol, 2009. 132(1): p. 47-58.

37. Kanellos, G. and M.C. Frame, Cellular functions of the ADF/cofilin family at a glance. J Cell Sci, 2016. 129(17): p. 3211-8.

38. Boyden, S., The chemotactic effect of mixtures of antibody and antigen on polymorphonuclear leucocytes. J Exp Med, 1962. 115: p. 453-66. 
39. Cioni, J.M., M. Koppers, and C.E. Holt, Molecular control of local translation in axon development and maintenance. Curr Opin Neurobiol, 2018. 51: p. 86-94.

40. Neriec, N. and P. Percipalle, Sorting mRNA Molecules for Cytoplasmic Transport and Localization. Front Genet, 2018. 9: p. 510.

41. Van Treeck, B. and R. Parker, Emerging Roles for Intermolecular RNARNA Interactions in RNP Assemblies. Cell, 2018. 174(4): p. 791-802.

42. Gehring, N.H., E. Wahle, and U. Fischer, Deciphering the mRNP Code: RNA-Bound Determinants of Post-Transcriptional Gene Regulation. Trends Biochem Sci, 2017. 42(5): p. 369-382.

43. Coppin, L., et al., Messenger RNA Life-Cycle in Cancer Cells: Emerging Role of Conventional and Non-Conventional RNA-Binding Proteins? Int J Mol Sci, 2018. 19(3).

44. Eliscovich, C. and R.H. Singer, RNP transport in cell biology: the long and winding road. Curr Opin Cell Biol, 2017. 45: p. 38-46.

45. Bergalet, J. and E. Lecuyer, The functions and regulatory principles of mRNA intracellular trafficking. Adv Exp Med Biol, 2014. 825: p. 57-96.

46. Jambhekar, A. and J.L. Derisi, Cis-acting determinants of asymmetric, cytoplasmic RNA transport. RNA, 2007. 13(5): p. 625-42.

47. Kislauskis, E.H., X. Zhu, and R.H. Singer, Sequences responsible for intracellular localization of beta-actin messenger RNA also affect cell phenotype. J Cell Biol, 1994. 127(2): p. 441-51.

48. Kislauskis, E.H., X. Zhu, and R.H. Singer, beta-Actin messenger RNA localization and protein synthesis augment cell motility. J Cell Biol, 1997. 136(6): p. 1263-70.

49. Yamagishi, M., Y. Shirasaki, and T. Funatsu, Size-dependent accumulation of mRNA at the leading edge of chicken embryo fibroblasts. Biochem Biophys Res Commun, 2009. 390(3): p. 750-4.

50. Hotz, M. and W.J. Nelson, Pumilio-dependent localization of mRNAs at the cell front coordinates multiple pathways required for chemotaxis. Nat Commun, 2017. 8(1): p. 1366.

51. Ross, A.F., et al., Characterization of a beta-actin mRNA zipcode-binding protein. Mol Cell Biol, 1997. 17(4): p. 2158-65. 
52. Huang, $X$., et al., Insulin-like growth factor $2 m R N A$-binding protein 1 (IGF2BP1) in cancer. J Hematol Oncol, 2018. 11(1): p. 88.

53. Rosenfeld, Y.B., et al., VICKZ1 enhances tumor progression and metastasis in lung adenocarcinomas in mice. Oncogene, 2019. 38(21): p. 4169-4181.

54. Stohr, N., et al., IGF2BP1 promotes cell migration by regulating MK5 and PTEN signaling. Genes Dev, 2012. 26(2): p. 176-89.

55. Vikesaa, J., et al., RNA-binding IMPs promote cell adhesion and invadopodia formation. EMBO J, 2006. 25(7): p. 1456-68.

56. Vainer, G., et al., A role for VICKZ proteins in the progression of colorectal carcinomas: regulating lamellipodia formation. J Pathol, 2008. 215(4): p. 445-56.

57. Wang, G., et al., IMP1 suppresses breast tumor growth and metastasis through the regulation of its target mRNAs. Oncotarget, 2016. 7(13): p. 15690-702.

58. Gu, W., et al., Regulation of local expression of cell adhesion and motility-related mRNAs in breast cancer cells by IMP1/ZBP1. J Cell Sci, 2012. 125(Pt 1): p. 81-91.

59. Huang, $\mathrm{H}$., et al., Recognition of RNA N(6)-methyladenosine by IGF2BP proteins enhances mRNA stability and translation. Nat Cell Biol, 2018. 20(3): p. 285-295.

60. Oleynikov, Y. and R.H. Singer, Real-time visualization of ZBP1 association with beta-actin $m R N A$ during transcription and localization. Curr Biol, 2003. 13(3): p. 199-207.

61. Pan, F., et al., ZBP2 facilitates binding of ZBP1 to beta-actin $m R N A$ during transcription. Mol Cell Biol, 2007. 27(23): p. 8340-51.

62. Patel, V.L., et al., Spatial arrangement of an RNA zipcode identifies mRNAs under post-transcriptional control. Genes Dev, 2012. 26(1): p. 43-53.

63. Chao, J.A., et al., ZBP1 recognition of beta-actin zipcode induces RNA looping. Genes Dev, 2010. 24(2): p. 148-58.

64. Farina, K.L., et al., Two ZBP1 KH domains facilitate beta-actin mRNA localization, granule formation, and cytoskeletal attachment. J Cell Biol, 2003. 160(1): p. 77-87. 
65. Song, T., et al., Specific interaction of KIF11 with ZBP1 regulates the transport of beta-actin mRNA and cell motility. J Cell Sci, 2015. 128(5): $p$. 1001-10.

66. Nathke, I.S., et al., The adenomatous polyposis coli tumor suppressor protein localizes to plasma membrane sites involved in active cell migration. J Cell Biol, 1996. 134(1): p. 165-79.

67. Mimori-Kiyosue, Y., N. Shiina, and S. Tsukita, Adenomatous polyposis coli (APC) protein moves along microtubules and concentrates at their growing ends in epithelial cells. J Cell Biol, 2000. 148(3): p. 505-18.

68. Harris, E.S. and W.J. Nelson, Adenomatous polyposis coli regulates endothelial cell migration independent of roles in beta-catenin signaling and cell-cell adhesion. Mol Biol Cell, 2010. 21(15): p. 2611-23.

69. Wen, Y., et al., EB1 and $A P C$ bind to mDia to stabilize microtubules downstream of Rho and promote cell migration. Nat Cell Biol, 2004. 6(9): p. 820-30.

70. Kroboth, K., et al., Lack of adenomatous polyposis coli protein correlates with a decrease in cell migration and overall changes in microtubule stability. Mol Biol Cell, 2007. 18(3): p. 910-8.

71. Yasuda, K., et al., The RNA-binding protein Fus directs translation of localized mRNAs in APC-RNP granules. J Cell Biol, 2013. 203(5): p. 737-46.

72. Chen, C., et al., Fused in Sarcoma: Properties, Self-Assembly and Correlation with Neurodegenerative Diseases. Molecules, 2019. 24(8).

73. Yasuda, K., et al., FUS inclusions disrupt RNA localization by sequestering kinesin-1 and inhibiting microtubule detyrosination. J Cell Biol, 2017. 216(4): p. 1015-1034.

74. Preitner, N., et al., APC is an RNA-binding protein, and its interactome provides a link to neural development and microtubule assembly. Cell, 2014. 158(2): p. 368-382.

75. Bellon, A. and F. Mann, Keeping up with advances in axon guidance. Curr Opin Neurobiol, 2018. 53: p. 183-191.

76. Leung, K.M., et al., Asymmetrical beta-actin mRNA translation in growth cones mediates attractive turning to netrin-1. Nat Neurosci, 2006. 9(10): p. 1247-56. 
77. Yao, J., et al., An essential role for beta-actin mRNA localization and translation in Ca2+-dependent growth cone guidance. Nat Neurosci, 2006. 9(10): p. 1265-73.

78. Wu, K.Y., et al., Local translation of RhoA regulates growth cone collapse. Nature, 2005. 436(7053): p. 1020-1024.

79. Piper, M., et al., Signaling mechanisms underlying Slit2-induced collapse of Xenopus retinal growth cones. Neuron, 2006. 49(2): p. 215-28.

80. Huttelmaier, S., et al., Spatial regulation of beta-actin translation by Srcdependent phosphorylation of ZBP1. Nature, 2005. 438(7067): p. 512-5.

81. Willett, M., et al., Translation initiation factors and active sites of protein synthesis co-localize at the leading edge of migrating fibroblasts. Biochem J, 2011. 438(1): p. 217-27.

82. Willett, M., et al., Localization of ribosomes and translation initiation factors to talin/beta3-integrin-enriched adhesion complexes in spreading and migrating mammalian cells. Biol Cell, 2010. 102(5): p. 265-76.

83. Katz, Z.B., et al., Mapping translation 'hot-spots' in live cells by tracking single molecules of mRNA and ribosomes. Elife, 2016. 5.

84. Paige, J.S., K.Y. Wu, and S.R. Jaffrey, RNA mimics of green fluorescent protein. Science, 2011. 333(6042): p. 642-6.

85. Sunbul, M. and A. Jaschke, Contact-mediated quenching for RNA imaging in bacteria with a fluorophore-binding aptamer. Angew Chem Int Ed Engl, 2013. 52(50): p. 13401-4.

86. Bertrand, E., et al., Localization of ASH1 mRNA particles in living yeast. Mol Cell, 1998. 2(4): p. 437-45.

87. Tyagi, S. and F.R. Kramer, Molecular beacons: probes that fluoresce upon hybridization. Nat Biotechnol, 1996. 14(3): p. 303-8.

88. Nelles, D.A., et al., Programmable RNA Tracking in Live Cells with CRISPR/Cas9. Cell, 2016. 165(2): p. 488-96.

89. Raj, A., et al., Imaging individual mRNA molecules using multiple singly labeled probes. Nat Methods, 2008. 5(10): p. 877-9.

90. Tsanov, N., et al., smiFISH and FISH-quant - a flexible single RNA detection approach with super-resolution capability. Nucleic Acids Res, 2016. 44(22): p. e165. 
91. Choi, H.M., et al., Programmable in situ amplification for multiplexed imaging of mRNA expression. Nat Biotechnol, 2010. 28(11): p. 1208-12.

92. Wang, F., et al., RNAscope: a novel in situ RNA analysis platform for formalin-fixed, paraffin-embedded tissues. J Mol Diagn, 2012. 14(1): p. 22-9.

93. Sinnamon, J.R. and K. Czaplinski, RNA detection in situ with FISHSTICs. RNA, 2014. 20(2): p. 260-6.

94. Lubeck, E., et al., Single-cell in situ RNA profiling by sequential hybridization. Nat Methods, 2014. 11(4): p. 360-1.

95. Eng, C.L., et al., Transcriptome-scale super-resolved imaging in tissues by RNA seqFISH. Nature, 2019. 568(7751): p. 235-239.

96. Halstead, J.M., et al., Translation. An RNA biosensor for imaging the first round of translation from single cells to living animals. Science, 2015. 347(6228): p. 1367-671.

97. Na, Y., et al., Real-Time Imaging Reveals Properties of GlutamateInduced Arc/Arg 3.1 Translation in Neuronal Dendrites. Neuron, 2016. 91(3): p. 561-73.

98. Yan, X., et al., Dynamics of Translation of Single mRNA Molecules In Vivo. Cell, 2016. 165(4): p. 976-89.

99. Wang, C., et al., Real-Time Imaging of Translation on Single mRNA Transcripts in Live Cells. Cell, 2016. 165(4): p. 990-1001.

100. Wu, B., et al., Translation dynamics of single mRNAs in live cells and neurons. Science, 2016. 352(6292): p. 1430-5.

101. Morisaki, T., et al., Real-time quantification of single RNA translation dynamics in living cells. Science, 2016. 352(6292): p. 1425-9.

102. tom Dieck, S., et al., Direct visualization of newly synthesized target proteins in situ. Nat Methods, 2015. 12(5): p. 411-4. 


\title{
Box 1. Visualizing posttranscriptional control of gene expression.
}

\author{
Detection of localized RNAs in living cells
}

- Aptamers (i) and hairpins (ii): tags inserted into the RNA sequence interact with fluorophores (i) or with RBPs fused to a fluorescent protein (FP) (ii). Improvements of these methods to enhance signal detection have included quenching (i) and nuclear targeting strategies (ii) of unbound fluorescent molecules [29, 84-86].

- Molecular beacons: hairpins recognizing specific RNA sequences are labeled with a quenched fluorophore. Upon binding, unfolding of the hairpin displaces the quencher and restores fluorophore signal [87].

- CRISPR/Cas9: sequence specific guide RNAs drive the binding of FP-tagged Cas9 to the target RNA molecule [88].

\section{Localized RNAs in fixed cells}

- smFISH (i) and smiFISH (ii): multiple individual oligonucleotide directly (i) or indirectly (ii) labeled with a fluorophore co-hybridize on a target RNA molecule [89, 90].

- FISH-STICs (i), bDNA (ii) and HCR (iii): single (i and iii) or double (ii) unlabeled oligonucleotides recognize RNA and are detected with the consecutive hybridization of amplifier and labeled detector probes ( $\mathrm{i}$ and ii) or hairpins that self-assemble into amplified polymers (iii). These systems provide signal improvements over smFISH and smiFISH at the expense of lengthier protocols [91-93].

- seqFISH: labeled oligonucleotides used in sequential rounds of hybridization allow multiplexed barcoding of RNA. An improved version involving pseudocoloring (seqFISH+) has shown enhanced resolution power [94, 95].

\section{Detection of local translation in living cells}

- TRICK: RNA molecules tagged with two different hairpin sequences, flanking the termination codon, are recognized by hairpin-specific RBPs fused to distinct FPs. During the first round of translation, ribosomes displace RBPs upstream of the termination codon leading to the loss of co-localization of the RBP signal [96].

- Luciferase fusions: Gaussia luciferase fused to the coding sequence of the protein of interest (POI) is expressed in cells cultured with substrate. Translation of the fusion protein results in a bioluminescent localized signal within seconds [97].

- SINAPS (i) and NCT (ii): tandem SunTag (i) or Flag (ii) epitopes tag the N-terminal of a POI. Nascent peptides are recognized by tag-specific antibody fragments labeled with FP or fluorophores, producing a rapidly detectable signal [98-101].

\section{Detection of local translation in fixed cells}

- Puro- (i) and FUNCAT-PLA (ii): metabolic incorporation of puromycin (i) or noncanonical amino acids (ii) labels nascent peptide chains during translation. Proximity detection with antibodies simultaneously targeting a particular POI and the metabolic modification allow the visualization of newly synthesized proteins in situ [102]. 


\section{Figure and table legends}

Figure 1. mRNA localization and local translation as modulators of cell migration

(A) Migratory cells acquire polarized morphologies, extending cell protrusions from their leading edges to sense and interact with the extracellular environment. As cells navigate through tissues, their movement is directed by, signaling factors, properties of the extracellular matrix and other cells. (B) mRNA localization and local translation are fundamental pathways in the spatial regulation of gene expression. Translating mRNAs into proteins within particular distal cell domains are hypothesized to confer advantages over the transport of proteins to these sites and to fine-tune biological responses: a) local translation of several proteins from one targeted mRNA molecule may be more costeffective than trafficking the same number of protein copies; b) local translation may increase the concentration of proteins enhancing regionalized effects; c) "new" locally synthesized proteins may undergo posttranslational modifications that renders them functionally distinct to "old" ones; d) complex formation may be facilitated by the spatial co-translation of its individual members; e) localized mRNA may act as a template reservoir readily available for protein production at sites where dynamic environmental stimuli elicit morphological changes such as membrane remodeling. Filled arrows indicate cell movement and mRNA/protein transport. Dashed arrows indicate biological processes.

\section{Figure 2. Mechanisms of mRNA transport and local translation.}

Two mechanisms orchestrating the localized presence of mRNAs in cell protrusions and their translation have been well studied in the context of cell migration. (A) ZBP1 transports $\beta$-actin in a translational silenced state along microfilaments and microtubules with the aid of motored proteins. At the leading edge, eF $1 \alpha$ acts as a scaffold protein capable of anchoring mRNAs to the actin network. Extracellular signaling activates Src kinase phosphorylation of ZBP1 to reduce its repressive activity, promoting $\beta$-actin release and translation. Synthesis of $\beta$-actin at the leading edge is preferentially associated to focal adhesion (FA) where it encourages adhesion and forward cell movement. (B) APC escorts mRNAs towards the plus $(+)$ end of detyrosinated microtubules in complexes containing FUS. Stiff substrates, induce cell contractility and the 
consequent microtubule detyrosination instruct mRNA localization via APC. Once they reach their destination, mRNAs trafficked are translated into proteins in a FUS-dependent manner. Filled arrows indicate mRNA transport. Dashed arrows indicate biological processes.

Table 1. Summary of published reports identifying protrusion-enriched mRNAs. 
Figure 2

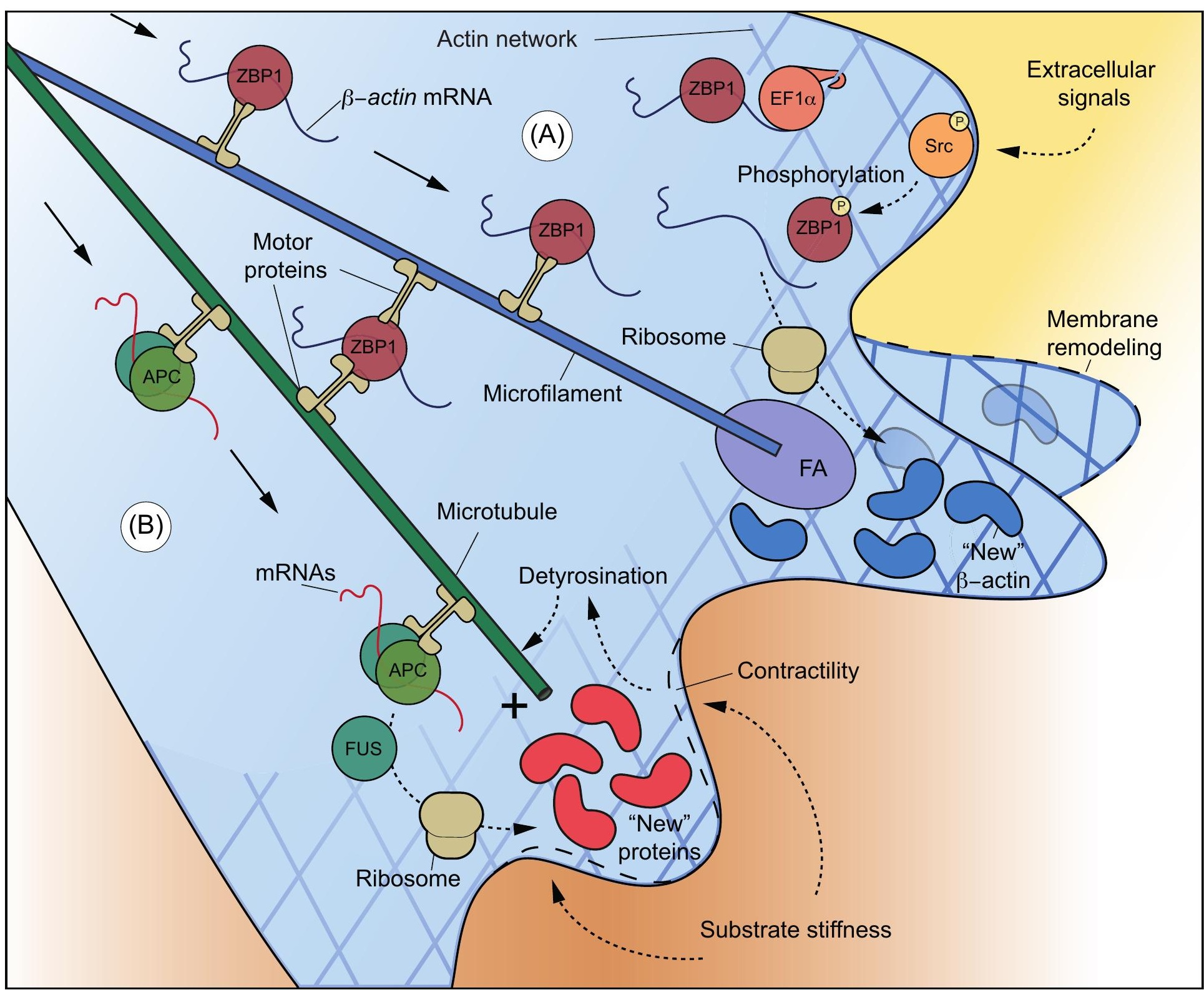




\section{Table 1}

\begin{tabular}{|c|c|c|c|c|}
\hline Cell type & $\begin{array}{l}\text { High-throughput } \\
\text { platform }\end{array}$ & $\begin{array}{l}\text { Fold change } \\
\text { threshold }^{\dagger}\end{array}$ & $\begin{array}{l}\text { Number } \\
\text { of mRNAs }\end{array}$ & Reference \\
\hline \multirow{3}{*}{$\begin{array}{c}\mathrm{NIH} / 3 \mathrm{~T} 3 \\
\text { (fibroblasts) }\end{array}$} & & 2.2 & $113^{a}$ & \multirow{2}{*}{ [11] } \\
\hline & 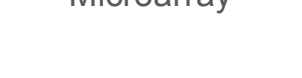 & 1.5 & $630^{\mathrm{b}}$ & \\
\hline & RNA-seq & 2 & 914 & [15] \\
\hline $\begin{array}{c}\text { MSV-MDCK-INV } \\
\text { (invasive variant MSV- } \\
\text { transformed epithelial } \\
\text { MDCK) }\end{array}$ & Microarray & 1.6 & 261 & [12] \\
\hline \multirow{2}{*}{$\begin{array}{c}\text { MDA-231 } \\
\text { (metastatic breast } \\
\text { cancer) }\end{array}$} & DRS & 1.6 & 709 & [13] \\
\hline & RNA-seq & $2^{\ddagger}$ & 1419 & [16] \\
\hline $\begin{array}{c}\text { Du145 [prostate] } \\
\text { MDA-231 [breast] } \\
\text { HT1080 [fibrosarcoma] } \\
\text { U251 [glioma] } \\
\text { U87 [glioma] } \\
\text { MDA-435 [breast] } \\
\text { (metastatic cancers) }\end{array}$ & Microarray & 1.6 & $384^{c}$ & [14] \\
\hline
\end{tabular}

${ }^{\dagger}$ Over cell bodies; ${ }^{\ddagger}$ post-publication; ${ }^{a}$ in response to LPA and ${ }^{b}$ fibronectin; ${ }^{c}$ overlap between cell types. 